\title{
S1
}

\section{Alkoxy-, Acyloxy and Bromomethylation of Resorcinarenes}

Sami Nummelin ${ }^{\dagger}$, Deszö Falabu ${ }^{\dagger}$, Alexander Shivanyuk ${ }^{\dagger, \dagger *}$, and Kari Rissanen ${ }^{\dagger *}$

Nanoscience Center, Department of Chemistry, University of Jyväskylä, P.O. Box 35, FIN-40351, Jyväskylä, Finland; Institute of Organic Chemistry, National Academy of Sciences of Ukraine, 02094 Kyiv 94, Murmanska str. 5, Ukraine.

1.1. E-mail: kari.rissanen@jyu.fi, shivan@bpci.kiev.ua

6a: $1 \mathrm{~g}$ (1.13 mmol) of $\mathbf{5 a}$ was dissolved in $30 \mathrm{ml}$ of acetanhydride and refluxed for 16 hours. The liquid was evaporated and the remaining solid triturated with $30 \mathrm{ml}$ of ethanol for 15 minutes. The solid was filtered and dried at room temperature. The compound was crystallised from a mixture of ethanol:dichloromethane $1: 1$.

${ }^{1} \mathrm{H}-\mathrm{NMR}\left(\mathrm{CDCl}_{3}, 303 \mathrm{~K}, 250 \mathrm{MHz}, \delta[\mathrm{ppm}]\right): 7.47$ (s, 2H, $\left.\mathrm{ArH}\right), 6.12(\mathrm{~s}, 2 \mathrm{H}, \mathrm{ArH}), 5.03(\mathrm{~s}, 4 \mathrm{H}$, $\left.\mathrm{ArCH}_{2} \mathrm{O}\right), 4.65\left(\mathrm{~s}, 4 \mathrm{H}, \mathrm{ArCH}_{2} \mathrm{O}\right), 4.14$ (q, 4H, $\left.\mathrm{CH}_{3} \mathrm{CHAr}_{2}\right), 2.37$ (s, 12H, $\left.\mathrm{COCH}_{3}\right), 2.10(\mathrm{~s}, 12 \mathrm{H}$, $\mathrm{COCH}_{3}$ ), 2.01 (s, 6H, $\mathrm{COCH}_{3}$ ), 1.87 (s, 6H, $\mathrm{COCH}_{3}$ ), 1.90 (q, 8H, $\mathrm{CH}_{2} \mathrm{CH}_{3}$ ), 1.47 (d, 12H, $\mathrm{Ar}_{2} \mathrm{CHCH}_{3}$ )

${ }^{13} \mathrm{C}-\mathrm{NMR}\left(\mathrm{CDCl}_{3}, 303 \mathrm{~K}, 62,9 \mathrm{MHz}, \delta[\mathrm{ppm}]\right): 170.4$ (CO), 168.9 (CO), $147.4(\mathrm{Ar}), 145.7$ (Ar), 137.4 (Ar), 133.6 (Ar), $127.9(\mathrm{ArH}), 125.1(\mathrm{ArH}), 123.3(\mathrm{Ar}), 121.1(\mathrm{Ar}), 56.7\left(\mathrm{ArCH}_{2} \mathrm{O}\right), 32.5\left(\mathrm{Ar}_{2} \mathrm{CHCH}_{3}\right)$, 20.7, 20.6, $20.2\left(\mathrm{CH}_{3}\right)$

DEPT-135 $\left(\mathrm{CDCl}_{3}, 303 \mathrm{~K}, 62,9 \mathrm{MHz}, \delta[\mathrm{ppm}]\right)$ : primary and tertiary carbons at: $127.9,125.1,32.5$, 20.7, 20.6, 20.2 secondary carbons at: 56.7

$\begin{array}{lc}\text { yield: } & 0.75 \mathrm{~g}, 57 \% \text { of crude } \\ \text { m.p.: } & >300^{\circ} \mathrm{C} \\ \text { molecular formula: } & \mathrm{C}_{60} \mathrm{H}_{64} \mathrm{O}_{24} \\ \text { m.w.: } & 1169.13 \\ \text { MS (ESI-TOF): } & 1191.36[\mathrm{M}+\mathrm{Na}]^{+} \\ \text {calculated composition } & \mathrm{C}(61.64 \%) \mathrm{H}(5.52 \%) \text { for } \mathrm{C}_{60} \mathrm{H}_{64} \mathrm{O}_{24} \\ \text { elemental analysis: } & \mathrm{C}(61.10 \%) \mathrm{H}(5.70 \%)\end{array}$

6b: $1 \mathrm{~g}(1.13 \mathrm{mmol})$ of $\mathbf{5 a}$ was dissolved in propionic anhydride and refluxed for 16 hours. The liquid was evaporated and a mixture of $30 \mathrm{ml}$ of methanol:dichloromethane 1:1 was added. Crystallisation started after 4 hours. The mother liquor was decanted and the remaining solid crystallised from diethyl ether:dichloromethane $1: 1$. 
${ }^{1} \mathrm{H}-\mathrm{NMR}\left(\mathrm{C}_{2} \mathrm{D}_{2} \mathrm{Cl}_{4}, 303 \mathrm{~K}, 250 \mathrm{MHz}, \delta[\mathrm{ppm}]\right): 7.47$ (s, 2H, $\left.\operatorname{Ar} H\right), 6.05$ (s, 2H, $\left.\operatorname{Ar} H\right), 4.97$ (s, 4H, $\mathrm{ArCH}_{2} \mathrm{O}$ ), 4.65 (s, $4 \mathrm{H}, \mathrm{ArCH}_{2} \mathrm{O}$ ), 4.14 (s, $4 \mathrm{H}, \mathrm{CH}_{3} \mathrm{CHAr}_{2}$ ), 2.69 (q, 8H, $\mathrm{COCH}_{2},{ }^{3} \mathrm{~J}=7.46 \mathrm{~Hz}$ ), 2.43 (q, $\left.8 \mathrm{H}, \mathrm{COCH}_{2},{ }^{3} \mathrm{~J}=7.16 \mathrm{~Hz}\right), 2.31\left(\mathrm{~m}, 4 \mathrm{H}, \mathrm{COCH}_{2},{ }^{3} \mathrm{~J}=7.61 \mathrm{~Hz}\right), 2.13\left(\mathrm{q}, 4 \mathrm{H}, \mathrm{COCH}_{2},{ }^{3} \mathrm{~J}=7.62 \mathrm{~Hz}\right), 1.47(\mathrm{~d}$, $\left.12 \mathrm{H}, \mathrm{Ar}_{2} \mathrm{CHCH}_{3},{ }^{3} \mathrm{~J}=6.7 \mathrm{~Hz}\right) 1.34\left(\mathrm{t}, 12 \mathrm{H}, \mathrm{CH}_{2} \mathrm{CH}_{3},{ }^{3} \mathrm{~J}=7.61 \mathrm{~Hz}\right), 1.15\left(\mathrm{t}, \mathrm{o}, \mathrm{CH}_{2} \mathrm{CH}_{3},{ }^{3} \mathrm{~J}=7.46 \mathrm{~Hz}\right), 1.08$ (t, o, $\left.\mathrm{CH}_{2} \mathrm{CH}_{3},{ }^{3} \mathrm{~J}=7.16 \mathrm{~Hz}\right), 1.00\left(\mathrm{t}, \mathrm{o}, \mathrm{CH}_{2} \mathrm{CH}_{3},{ }^{3} \mathrm{~J}=7.46 \mathrm{~Hz}\right)$

${ }^{13} \mathrm{C}-\mathrm{NMR}\left(\mathrm{C}_{2} \mathrm{D}_{2} \mathrm{Cl}_{4}, 303 \mathrm{~K}, 62,9 \mathrm{MHz}, \delta[\mathrm{ppm}]\right): 173.6,173.5,173.2(\mathrm{CO}) 171.3$ (br, CO), 147.2, 145.3, 136.9, 133.1, 127.2, 124.8, 122.9, 120.7 (Ar-s), $56.3\left(\mathrm{ArCH}_{2} \mathrm{O}\right), 32.0\left(\mathrm{Ar}_{2} \mathrm{CHCH}_{3}\right), 27.19,27.10$, 26.97, $26.55\left(\mathrm{CH}_{2}\right), 19.8\left(\mathrm{CHCH}_{3}\right), 9.0,8.9,8.7,8.5\left(\mathrm{CH}_{2 \mathrm{C}} \mathrm{H}_{3}\right)$

DEPT-135 $\left(\mathrm{C}_{2} \mathrm{D}_{2} \mathrm{Cl}_{4}, 303 \mathrm{~K}, 62.9 \mathrm{MHz}, \delta[\mathrm{ppm}]\right)$ : primary and tertiary carbons at $127.2,124.8,32.0$, $19.8,9.0,8.9,8.7,8.5$, secondary carbons at $56.2,27.19,27.10,26.97,26.55$

yield: $\quad 0.4 \mathrm{~g}, 27 \%$ after crystallisation

m.p.: $\quad 223^{\circ} \mathrm{C}$

molecular formula: $\quad \mathrm{C}_{72} \mathrm{H}_{88} \mathrm{O}_{24}$

m.w.: $\quad 1337.45$

MS (ESI-TOF): $\quad 1359.19[\mathrm{M}+\mathrm{Na}]^{+}$

calculated composition $\quad \mathrm{C}(64.66 \%) \mathrm{H}(6.63 \%)$ for $\mathrm{C}_{72} \mathrm{H}_{88} \mathrm{O}_{24}$

elemental analysis: $\quad \mathrm{C}(64.56 \%) \mathrm{H}(6.72 \%)$

6c: $1.5 \mathrm{~g}(2.5 \mathrm{mmol})$ of $\mathbf{5 b}$ was refluxed in $30 \mathrm{ml}$ of acetic anhydride for 4 hours. The liquid was evaporated and the oily solid triturated with $30 \mathrm{ml}$ of ethanol twice. The remaining solid was filtered and crystallised from ethyl acetate. Second crystallisation provided crystals suitable for X-ray analysis. ${ }^{1} \mathrm{H}-\mathrm{NMR}\left(\mathrm{CDCl}_{3}, 303 \mathrm{~K}, 250 \mathrm{MHz}, \delta[\mathrm{ppm}]\right): \sim 8-6(\mathrm{br}, \mathrm{ArH}), 4.86\left(\mathrm{~s}, 8 \mathrm{H}, \mathrm{ArCH}_{2} \mathrm{O}\right), 3.93$ (t, 4H, $\left.\mathrm{RCHAr}_{2}\right), 2.26\left(\mathrm{~s}, 24 \mathrm{H}, \mathrm{COCH}_{3}\right), 1.97\left(\mathrm{~s}, 12 \mathrm{H}, \mathrm{COCH}_{3}\right), 1.9\left(\mathrm{~m}, 8 \mathrm{H}, \mathrm{CH}_{2} \mathrm{CH}_{3}\right), 0.93(\mathrm{t}, 12 \mathrm{H}$, $\left.\mathrm{CHCH}_{2} \mathrm{CH}_{3}\right)$

${ }^{1} \mathrm{H}-\mathrm{NMR}\left(\mathrm{DMSO}_{6}, 303 \mathrm{~K}, 500 \mathrm{MHz}, \delta[\mathrm{ppm}]\right): ~ 8-7$ (br, $\mathrm{ArH}$ ), 4.83 (s, 8H, $\mathrm{ArCH}_{2} \mathrm{O}$ ), 3.78 (t, $4 \mathrm{H}$, $\left.\mathrm{RCHAr}_{2}\right), 2.35\left(\mathrm{~s}, 24 \mathrm{H}, \mathrm{COCH}_{3}\right), 2.05\left(\mathrm{~m}, 8 \mathrm{H}, \mathrm{CH}_{2} \mathrm{CH}_{3}\right), 1.95\left(\mathrm{~s}, 12 \mathrm{H}, \mathrm{COCH}_{3}\right), 0.81(\mathrm{t}, 12 \mathrm{H}$, $\mathrm{CHCH}_{2} \mathrm{CH}_{3}$ )

1'H-NMR (DMSO-D6, $348 \mathrm{~K}, 500 \mathrm{MHz}, \delta[\mathrm{ppm}]): 7.22$ (s, 4H ArH), 4.81 (s, 8H, $\mathrm{ArCH}_{2} \mathrm{O}$ ), 3.87 (t, 4H, RCHAr $)_{2}, 2.33\left(\mathrm{~s}, 24 \mathrm{H}, \mathrm{COCH}_{3}\right), 2.02\left(\mathrm{~m}, 8 \mathrm{H}, \mathrm{CH}_{2} \mathrm{CH}_{3}\right), 1.93\left(\mathrm{~s}, 12 \mathrm{H}, \mathrm{COCH}_{3}\right), 0.86(\mathrm{t}, 12 \mathrm{H}$, $\mathrm{CHCH}_{2} \mathrm{CH}_{3}$ )

${ }^{13} \mathrm{C}-\mathrm{NMR}\left(\mathrm{CDCl}_{3}, 303 \mathrm{~K}, 62,9 \mathrm{MHz}, \delta[\mathrm{ppm}]\right): 170.5(\mathrm{CO}), 168.7$ (CO), 160-120 (br, Ar-s), 56.9 $\left(\mathrm{ArCH}_{2} \mathrm{O}\right), 39.6\left(\mathrm{Ar}_{2} \mathrm{CH}\right), 27.4\left(\mathrm{CHCH}_{2} \mathrm{CH}_{3}\right), 20.8,20.6\left(\mathrm{COCH}_{3}\right), 12.4\left(\mathrm{CHCH}_{2} \mathrm{CH}_{3}\right)$. 
S3

${ }^{13} \mathrm{C}-\mathrm{NMR}\left(\mathrm{CDCl}_{3}, 303 \mathrm{~K}, 125,7 \mathrm{MHz}, \delta[\mathrm{ppm}]\right): 170.3(\mathrm{CO}), 168.4(\mathrm{CO}), 146.5 ; 135.6 ; 126.4 ; 122.3$ (br, $\mathrm{Ar}), 56.7\left(\mathrm{ArCH}_{2} \mathrm{O}\right), 39.4\left(\mathrm{Ar}_{2} \mathrm{CH}\right), 27.1\left(\mathrm{CHCH}_{2} \mathrm{CH}_{3}\right), 20.5,20.3\left(\mathrm{COCH}_{3}\right), 12.8\left(\mathrm{CHCH}_{2} \mathrm{CH}_{3}\right)$

${ }^{13} \mathrm{C}-\mathrm{NMR}\left(\mathrm{DMSO}_{6}, 348 \mathrm{~K}, 125,7 \mathrm{MHz}, \delta[\mathrm{ppm}]\right): 169.1$ (CO), 167.9 (CO), 146.0; 133.2; 126.7; $121.2(\mathrm{Ar}), 55.7\left(\mathrm{ArCH}_{2} \mathrm{O}\right), 38.3\left(\mathrm{Ar}_{2} \mathrm{CH}\right), 26.0\left(\mathrm{CHCH}_{2} \mathrm{CH}_{3}\right), 19.7,19.6\left(\mathrm{COCH}_{3}\right), 11.8\left(\mathrm{CHCH}_{2} \mathrm{CH}_{3}\right)$ DEPT-135 $\left(\mathrm{CDCl}_{3}, 303 \mathrm{~K}, 62,9 \mathrm{MHz}, \delta[\mathrm{ppm}]\right)$ : primary and tertiary carbons at: not visible $(A r \mathrm{H})$, 39.4, 20.5, 20.3, 12.8, secondary carbons at: 567, 27.1 .

$\begin{array}{lc}\text { yield: } & 1.70 \mathrm{~g}, 52 \% \text { after crystallisation } \\ \text { m.p.: } & 224.3^{\circ} \mathrm{C} \\ \text { molecular formula: } & \mathrm{C}_{64} \mathrm{H}_{72} \mathrm{O}_{24} \\ \text { m.w.: } & 1225.24 \\ \text { MS (ESI-TOF): } & 1247.22[\mathrm{M}+\mathrm{Na}]^{+} \\ \text {MS (MALDI-TOF): } & 1248.49[\mathrm{M}+\mathrm{Na}]^{+} \\ \text {calculated composition } & \mathrm{C}(59.59 \%) \mathrm{H}(5.69 \%) \text { for } \mathrm{C}_{64} \mathrm{H}_{72} \mathrm{O}_{24} * \mathrm{CH}_{2} \mathrm{Cl}_{2} \\ \text { elemental analysis: } & \mathrm{C}(59.36 \%) \mathrm{H}(5.87 \%)\end{array}$

6d: $1.5 \mathrm{~g}(2.5 \mathrm{mmol})$ of $\mathbf{5 b}$ was dissolved in $30 \mathrm{ml}$ of propionic anhydride and refluxed for 24 hours. The liquid was evaporated and $20 \mathrm{ml}$ of methanol was added to the resulted oil, which solidified in 3 days. The product was washed with ethanol several times until a brown oil could be separated. The resulted amber-coloured solid was crystallised from ethanol affording colourless crystals.

${ }^{1} \mathrm{H}-\mathrm{NMR}\left(\mathrm{C}_{2} \mathrm{D}_{2} \mathrm{Cl}_{4}, 303 \mathrm{~K}, 250 \mathrm{MHz}, \delta[\mathrm{ppm}]\right): 7.38$ (br, s, 2H, $\mathrm{ArH}$ ), 6.03 (br, s, 2H, $\mathrm{ArH}$ ), 4.95 (br, s, $4 \mathrm{H}, \mathrm{ArCH}_{2} \mathrm{O}$ ), 4.65 (br, s, 4H, $\mathrm{ArCH}_{2} \mathrm{O}$ ), 3.93 (s, 4H, $\mathrm{CH}_{3} \mathrm{CHAr}_{2}$ ), 2.7-1.6 (m, o, 32H, $\mathrm{OCOCH}_{2}$, $\left.\mathrm{CH}_{2} \mathrm{OCOCH}_{2}, \mathrm{CHCH}_{2} \mathrm{CH}_{3}\right), 1.5-0.8$ (m, o, $48 \mathrm{H}, \mathrm{OCOCH}_{2} \mathrm{CH}_{3}, \mathrm{CH}_{2} \mathrm{OCOCH}_{2} \mathrm{CH}_{3}, \mathrm{Ar}_{2} \mathrm{CHCH}_{2} \mathrm{CH}_{3}$ ) ${ }^{1} \mathrm{H}-\mathrm{NMR}\left(\mathrm{C}_{2} \mathrm{D}_{2} \mathrm{Cl}_{4}, 373 \mathrm{~K}, 250 \mathrm{MHz}, \delta[\mathrm{ppm}]\right): 6.8(\mathrm{br}, \mathrm{s}, 4 \mathrm{H}, \mathrm{ArH}), 4.89$ (s, 8H, $\left.\mathrm{ArCH}_{2} \mathrm{O}\right), 4.04(\mathrm{~s}, 4 \mathrm{H}$, $\mathrm{CH}_{3} \mathrm{CHAr}_{2}$ ), 2.58 (q, 16H, OCOCH${ }_{2}$ ), 2.26 (q, 8H, $\mathrm{CH}_{2} \mathrm{OCOCH}_{2}$ ), 1.95 (m, 8H, $\left.\mathrm{CHCH}_{2} \mathrm{CH}_{3}\right), 1.26$ (t, $\left.24 \mathrm{H}, \mathrm{OCOCH}_{2} \mathrm{CH}_{3}\right), 1.14\left(\mathrm{t}, 12 \mathrm{H}, \mathrm{CH}_{2} \mathrm{OCOCH}_{2} \mathrm{CH}_{3}\right), 0.98$ (t, $\left.12 \mathrm{H}, \mathrm{Ar}_{2} \mathrm{CHCH}_{2} \mathrm{CH}_{3}\right)$

${ }^{13} \mathrm{C}-\mathrm{NMR}\left(\mathrm{C}_{2} \mathrm{D}_{2} \mathrm{Cl}_{4}, 303 \mathrm{~K}, 250 \mathrm{MHz}, \delta[\mathrm{ppm}]\right): 173.5(\mathrm{CO}), 172.0$ (br, CO), $148.0(\mathrm{br}, \mathrm{ArO}), 145.2(\mathrm{br}$, ArO), 135.8 (br, Ar), 130.8 (br, Ar), 127.6 (br, Ar), 125.3 (br, Ar), 123.1 (br, Ar), 120.9 (br, Ar), 56.4 (br, $\mathrm{ArCH}_{2} \mathrm{O}$ ), $39.1(\mathrm{ArCH}), 27.0,26.6$ (br, o, $\left.\mathrm{COCH}_{2}, \mathrm{COCH}_{2}, \mathrm{CHCH}_{2}\right), 12.2\left(\mathrm{CHCH}_{2} \mathrm{CH}_{3}\right), 8.8,8.5$ (br, o, $\mathrm{COCH}_{2} \mathrm{CH}_{3}, \mathrm{COCH}_{2} \mathrm{CH}_{3}$ ) Boat-boat transition is at $303 \mathrm{~K}$.

${ }^{13} \mathrm{C}-\mathrm{NMR}\left(\mathrm{C}_{2} \mathrm{D}_{2} \mathrm{Cl}_{4}, 373 \mathrm{~K}, 250 \mathrm{MHz}, \delta[\mathrm{ppm}]\right): 173.1(\mathrm{CO}), 171.3(\mathrm{br}, \mathrm{CO}), 147.0(\mathrm{br}, A r \mathrm{O}), 133.1$ (br, Ar), $126.8(\mathrm{ArH}), 122.3(\mathrm{Ar}), 56.4\left(\mathrm{br}, \mathrm{ArCH}_{2} \mathrm{O}\right), 39.5(\mathrm{ArCH}), 27.0,26.8,26.6\left(\mathrm{o}, \mathrm{COCH}_{2}, \mathrm{COCH}_{2}\right.$, $\left.\mathrm{CHCH}_{2}\right), 12.2\left(\mathrm{CHCH}_{2} \mathrm{CH}_{3}\right), 8.5,8.4\left(\mathrm{COCH}_{2} \mathrm{CH}_{3}, \mathrm{COCH}_{2} \mathrm{CH}_{3}\right)$ Boat-boat interconversion is faster than the NMR time scale at $373 \mathrm{~K}$.

DEPT-135 $\left(\mathrm{C}_{2} \mathrm{D}_{2} \mathrm{Cl}_{4}, 373 \mathrm{~K}, 250 \mathrm{MHz}, \delta[\mathrm{ppm}]\right)$ : primary and tertiary carbons at: $126.8,39.5,12.2,8.5$, 8.4, secondary carbons at: $56.4,27.0,26.8,26.6$

yield:

$0.66 \mathrm{~g}, 19 \%$ after crystallisation

m.p.: $196.2^{\circ} \mathrm{C}$ 
molecular formula:

$\mathrm{C}_{76} \mathrm{H}_{96} \mathrm{O}_{24}$

S5

m.w.:

1393.56

MS (ESI-TOF):

$1415.38[\mathrm{M}+\mathrm{Na}]^{+}$

calculated composition

$\mathrm{C}(65.50 \%) \mathrm{H}(6.94 \%)$ for $\mathrm{C}_{76} \mathrm{H}_{96} \mathrm{O}_{24}$

elemental analysis:

$\mathrm{C}(65.27 \%) \mathrm{H}(7.00 \%)$

6e: $2 \mathrm{~g}(1.8 \mathrm{mmol})$ of $\mathbf{5 c}$ was dissolved in $30 \mathrm{ml}$ of acetic anhydride and refluxed for two days. The liquid was evaporated and a mixture of $40 \mathrm{ml}$ of toluene:ethanol 1:1 was added to the residue and evaporated twice. The product was crystallised from $\mathrm{CH}_{2} \mathrm{Cl}_{2}$ :diethyl ether 1:9. The solid was first dissolved in $\mathrm{CH}_{2} \mathrm{Cl}_{2}$ then this solution mixed with 9 parts of diethyl ether relative to $\mathrm{CH}_{2} \mathrm{Cl}_{2}$.

$\underline{{ }^{1} \mathrm{H}-\mathrm{NMR}}\left(\mathrm{CDCl}_{3}, 303 \mathrm{~K}, 250 \mathrm{MHz}, \delta[\mathrm{ppm}]\right): 7.46$ (o, $\left.\mathrm{CDCl}_{3}, \mathrm{~s}, 2 \mathrm{H}, \mathrm{ArH}\right), 6.10$ (s, 2H, $\left.\mathrm{ArH}\right), 4.99$ (s, o, $\left.4 \mathrm{H}, \mathrm{OCH}_{2} \mathrm{Ar}\right), 4.70$ (s, o, 4H, OCH $\mathrm{O}_{2} \mathrm{Ar}$ ), 4.02 (t, 4H, $\mathrm{CH}_{3} \mathrm{CHAr}_{2}$ ), 2.30-1.50 (o, 44H, $\mathrm{COCH}_{3}$, $\left.\mathrm{CHCH}_{2} \mathrm{CH}_{2}\right), 1.25\left(\mathrm{o}, 24 \mathrm{H},\left(\mathrm{CH}_{2}\right)_{3} \mathrm{CH}_{3}\right), 0.85\left(\mathrm{t}, 12 \mathrm{H} \mathrm{CH}_{2} \mathrm{CH}_{3}\right)$

${ }^{1} \mathrm{H}-\mathrm{NMR}\left(\mathrm{C}_{2} \mathrm{D}_{2} \mathrm{Cl}_{4}, 373 \mathrm{~K}, 250 \mathrm{MHz}, \delta[\mathrm{ppm}]\right): 6.73$ (s, br, 4H, $\left.\mathrm{ArH}\right) 4.81\left(\mathrm{~s}, 8 \mathrm{H}, \mathrm{OCH}_{2} \mathrm{Ar} 4.06(\mathrm{t}, 4 \mathrm{H}\right.$, $\left.\mathrm{CH}_{3} \mathrm{CHAr}_{2}\right), 2.18\left(\mathrm{~s}, 24 \mathrm{H}, \mathrm{COCH}_{3}\right), 1.92\left(\mathrm{~s}, 12 \mathrm{H}, \mathrm{COCH}_{3}\right), 1.83\left(\mathrm{~m}, 8 \mathrm{H}, \mathrm{CHCH}_{2} \mathrm{CH}_{2}\right), 1.26(\mathrm{o}, 24 \mathrm{H}$, $\left.\left(\mathrm{CH}_{2}\right)_{3} \mathrm{CH}_{3}\right), 0.85\left(\mathrm{t}, 12 \mathrm{H}, \mathrm{CH}_{2} \mathrm{CH}_{3}\right)$

奋-NMR (DMSO-D, 373 K, $250 \mathrm{MHz}, \delta[\mathrm{ppm}]): 7.11$ (s, 4H, ArH) 4.82 (s, 8H, OCH $\mathrm{Ar} 4.03$ (t, 4H, $\mathrm{CH}_{3} \mathrm{CHAr}_{2}$ ), $2.28\left(\mathrm{~s}, 24 \mathrm{H}, \mathrm{COCH}_{3}\right), 1.99\left(\mathrm{~m}, 8 \mathrm{H}, \mathrm{CHCH}_{2} \mathrm{CH}_{2}\right), 1.94\left(\mathrm{~s}, 12 \mathrm{H}, \mathrm{COCH}_{3}\right), 1.30(\mathrm{o}, 24 \mathrm{H}$, $\left.\left(\mathrm{CH}_{2}\right)_{3} \mathrm{CH}_{3}\right), 0.87\left(\mathrm{t}, 12 \mathrm{H}, \mathrm{CH}_{2} \mathrm{CH}_{3}\right)$

${ }^{13} \mathrm{C}-\mathrm{NMR}\left(\mathrm{CDCl}_{3}, 323 \mathrm{~K}, 62.9 \mathrm{MHz}, \delta[\mathrm{ppm}]\right): 169.4$ (CO), 167.4 (CO), not visible (Ar-s), 56.2 $\left(\mathrm{ArCH} \mathrm{H}_{2} \mathrm{O}\right), 36.9\left(\mathrm{Ar}_{2} \mathrm{CHCH}_{2}\right), 33.1,31.0,27.1,21.7\left(\left(\mathrm{CH}_{2}\right)_{4} \mathrm{CH}_{3}\right), 19.7,19.5\left(\mathrm{COCH}_{3}\right), 13.1\left(\mathrm{CH}_{2} \mathrm{CH}_{3}\right)$ ${ }^{13} \mathrm{C}-\mathrm{NMR}\left(\mathrm{DMSO}-\mathrm{D}_{6}, 373 \mathrm{~K}, 250 \mathrm{MHz}, \delta[\mathrm{ppm}]\right): 168.9$ (CO), 167.6 (CO), 145.8 (Ar), 133.1 (Ar), $126.5(\mathrm{ArH}), 121.2(\mathrm{Ar}), 55.6\left(\mathrm{ArCH}_{2} \mathrm{O}\right), 36.3\left(\mathrm{Ar}_{2} \mathrm{CHCH}_{2}\right), 32.7,30.5,26.6,21.2,\left(\left(\mathrm{CH}_{2}\right)_{4} \mathrm{CH}_{3}\right), 19.47$, $19.40\left(\mathrm{COCH}_{3}\right), 12.8\left(\mathrm{CH}_{2} \mathrm{CH}_{3}\right)$

DEPT-135 (DMSO-D $6,373 \mathrm{~K}, 250 \mathrm{MHz}, \delta[\mathrm{ppm}])$ : primary and tertiary carbons at: 126.5, 36.3, 19.47, 19.40, secondary carbons at: 55.6, 32.7, 30.5, 26.6, 21.2

yield:

$0.57 \mathrm{~g}, 23 \%$ after crystallization.

m.p.: $192.6^{\circ} \mathrm{C}$

molecular formula: $\mathrm{C}_{76} \mathrm{H}_{96} \mathrm{O}_{24}$

m.w.: 1393.56

MS (ESI-TOF): $1415.44[\mathrm{M}+\mathrm{Na}]^{+}$ calculated composition $\mathrm{C}(65.50 \%) \mathrm{H}(6.94 \%)$ for $\mathrm{C}_{76} \mathrm{H}_{96} \mathrm{O}_{24}$ elemental analysis: $\mathrm{C}(65.23 \%) \mathrm{H}(6.88 \%)$ 


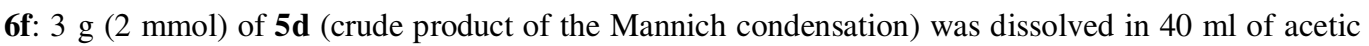
anhydride and refluxed for 3 days. The solvent was evaporated and $50 \mathrm{ml}$ of ethanol was added to the oily residue. Water was dropped slowly into the mixture under vigorous stirring until the transparent solution turned to slightly turbid. The stirring was continued for overnight meanwhile a white precipitation formed. It was filtered, crystallised from $\mathrm{CH}_{2} \mathrm{Cl}_{2}$ :diethyl ether 1:9 and dried at room temperature.

${ }^{1} \mathrm{H}-\mathrm{NMR}\left(\mathrm{CDCl}_{3}, 303 \mathrm{~K}, 250 \mathrm{MHz}, \delta[\mathrm{ppm}]\right): 7.3\left(\mathrm{o}, \mathrm{CDCl}_{3}\right.$, br, $\left.\mathrm{Ar} H\right), 6.1$ (br, $\left.\mathrm{Ar} H\right) 4.9$ (br, about coalescence $8 \mathrm{H}, \mathrm{OCH}_{2} \mathrm{Ar}$ ), 4.00 (t, 4H, $\mathrm{CH}_{2} \mathrm{CHAr}_{2}$ ), 2.24 (s, br, 24H, $\left.\mathrm{COCH}_{3}\right), 1.95$ (s, 12H, $\mathrm{COCH}_{3}$ ), $1.84\left(\mathrm{~m}, 8 \mathrm{H}, \mathrm{CH}_{2} \mathrm{CH}_{3}\right), 1.24\left(\mathrm{o}, 72 \mathrm{H}, \mathrm{CH}_{2}-\mathrm{s}\right), 0.87$ (t, $12 \mathrm{~h}, \mathrm{CH}_{2} \mathrm{CH}_{3}$ )

${ }^{1} \mathrm{H}-\mathrm{NMR}\left(\mathrm{C}_{2} \mathrm{D}_{2} \mathrm{Cl}_{4}, 373 \mathrm{~K}, 250 \mathrm{MHz}, \delta[\mathrm{ppm}]\right): 6.81$ (s, br, $\mathrm{Ar} H$ ), 4.90 (s, 8H, OCH $\left.\mathrm{Or}_{2} \mathrm{Ar}\right), 4.14$ (t, 4H, $\mathrm{CH}_{2} \mathrm{CHAr}_{2}$ ), 2.27 (s, br, 24H, $\left.\mathrm{COCH}_{3}\right), 2.00\left(\mathrm{~s}, 12 \mathrm{H}, \mathrm{COCH}_{3}\right), 1.94\left(\mathrm{~m}, 8 \mathrm{H}, \mathrm{CH}_{2} \mathrm{CH}_{3}\right), 1.33$ (o, 72H, $\mathrm{CH}_{2}$-s), 0.94 (t, $12 \mathrm{~h}, \mathrm{CH}_{2} \mathrm{CH}_{3}$ )

${ }^{13} \mathrm{C}-\mathrm{NMR}\left(\mathrm{CDCl}_{3}, 303 \mathrm{~K}, 62.9 \mathrm{MHz}, \delta[\mathrm{ppm}]\right): 170.5,(\mathrm{CO}), 168.6$, $(\mathrm{CO})$, not visible $(\mathrm{Ar}$-s), 56.8 ( $\left.\mathrm{ArCH}_{2} \mathrm{O}\right), 37.6\left(\mathrm{Ar}_{2} \mathrm{CHCH}_{2}\right), 34.2\left(\mathrm{CHCH}_{2} \mathrm{CH}_{2}\right), 32.0,29.9,29.86,29.83,29.5,28.4,\left(\mathrm{CH}_{2}-\mathrm{s}\right), 20.7$, $20.5\left(\mathrm{COCH}_{3}\right), 14.2\left(\mathrm{CH}_{2} \mathrm{CH}_{3}\right)$, (two alkylmethylene signals are not visible.)

${ }^{13} \mathrm{C}-\mathrm{NMR}\left(\mathrm{C}_{2} \mathrm{D}_{2} \mathrm{Cl}_{4}, 373 \mathrm{~K}, 62.9 \mathrm{MHz}, \delta[\mathrm{ppm}]\right): 169.6(\mathrm{CO}), 167.6,(\mathrm{CO}), 146.7(\mathrm{Ar}), 133.5(\mathrm{Ar})$, 126.6 (ArH), $122.2(\mathrm{Ar}), 56.7\left(\mathrm{ArCH}_{2} \mathrm{O}\right), 37.7\left(\mathrm{Ar}_{2} \mathrm{CHCH}_{2}\right), 33.9\left(\mathrm{CHCH}_{2} \mathrm{CH}_{2}\right), 31.5,29.4,29.3,29.2$, 28.8, 27.9, $22.2\left(\mathrm{CH}_{2}-\mathrm{s}\right), 20.0,19.8\left(\mathrm{COCH}_{3}\right), 13.5\left(\mathrm{CH}_{2} \mathrm{CH}_{3}\right)$, (one alkylmethylene signal is not visible.)

DEPT-135 $\left(\mathrm{C}_{2} \mathrm{D}_{2} \mathrm{Cl}_{4}, 373 \mathrm{~K}, 62.9 \mathrm{MHz}, \delta[\mathrm{ppm}]\right)$ : primary and tertiary carbons at: $126.6,37.7,20.0$, 19.8, 13.5, secondary carbons at: $56.7,33.9,31.5,29.4,29.3,29.2,28.8,27.9,22.2$

yield: $0.69 \mathrm{~g}, 19 \%$ after crystallisation

m.p.: $85.4^{\circ} \mathrm{C}$

molecular formula:

$\mathrm{C}_{100} \mathrm{H}_{144} \mathrm{O}_{24}$

m.w.:

1730.19

MS (ESI-TOF):

$1752.77[\mathrm{M}+\mathrm{Na}]^{+}$

calculated composition

$\mathrm{C}(68.84 \%) \mathrm{H}(8.47 \%)$ for $\mathrm{C}_{100} \mathrm{H}_{144} \mathrm{O}_{24} * \mathrm{MeOH}$

elemental analysis:

$\mathrm{C}(68.80 \%) \mathrm{H}(8.64 \%)$ 